\title{
Renormalization of Feynman Amplitudes and Parametric Integral Representation
}

\author{
M. C. Bergère ${ }^{\star}$ and J. B. Zuber* \\ Service de Physique Théorique, Centre d'Etudes Nucléaires de Saclay, \\ Gif-sur-Yvette, France
}

Received July 10, 1973

\begin{abstract}
A new substraction formula is presented to renormalize Feynman amplitudes written in Schwinger's integral representation.

The substractions are generated by an operator acting on the integrand, which only depends on the total number of internal lines but is completely independent of the structure of the graph.

This formulation is also valid for non-renormalizable theories and is shown to reduce to Zimmermann's $R$-operation for scalar theories. It satisfies in any case Bogoliubov's recursive formula and yields an explicit tool for actual computations of renormalized Feynman amplitudes with a minimal number of substractions.
\end{abstract}

\section{Introduction}

A procedure for extracting finite parts from divergent Feynman amplitudes with the requirements of Lorentz covariance, causality and unitarity was given several years ago by Bogoliubov and Parasiuk [1]. They introduced a substraction procedure, the so-called $R$ operation, defined recursively over the graphs and the subgraphs and they proved this method to be equivalent to the addition of infinite counter terms in the Lagrangian. The proof of the B.P. theorem was then completed by Hepp [2] and recently Epstein and Glaser [3] reformulated the problem as a decomposition of distributions in retarded and advanced parts.

On the other hand, using the parametric integral representation [6], Appelquist [4] was able to give in closed form the value of a substracted Feynman amplitude. He proved in the case of renormalizable theories that his substraction scheme satisfies the B.P.H. recursive prescription. This proof was established by decomposing the families of subgraphs into forests. Later, this was proved by Zimmermann [5] to be, in the momentum space, the general solution to the recursion of B.P.H. even in the case of non-renormalizable theories.

* Attachê de Recherches CNRS. 
However, these explicit substraction formulae given by Appelquist and Zimmermann, involved crucially the knowledge of the topology of the graph.

In this paper, we have generalized Appelquist's result to any theory, renormalizable or not, and found an operator generating the substractions, which is completely independent of the topology of the graph except for the total number of internal lines. The renormalized Feynman amplitude is proved to be equal to Zimmermann's finite part in the case of scalar theories but differs from it by a finite renormalization when higher spins are involved.

This paper is organized as follows:

For the sake of simplicity, the three first sections deal only with spinless theories without derivative couplings.

In Section II we gather some known results about the parametric integral representation; we introduce in Section III our definition of the renormalized amplitude and prove the equivalence with the decomposition into forests; then Section IV is devoted to Zimmermann's formalism and its translation into parametric language. Finally in Section V, the different results are extended to the case of theories with spin and derivative couplings. In Section VI, we summarize our results and indicate some possible applications. The first Appendix describes the various properties of our substraction operator which is based on the definition of a generalized Taylor expansion. Then Appendix II and III explicit the complete proof of two intricate theorems (the first one was actually established by Appelquist [4]).

\section{Parametric Integral Representation of Feynman Amplitude}

For the sake of simplicity we first present the parametric integral representation for graphs associated with a spinless theory with non derivative but possibly non-renormalizable couplings.

To any connected Feynman graph with $l$ lines and $n$ vertices corresponds the following Feynman amplitude.

$$
I=\int \prod_{a=1}^{l} d^{D} k_{a} \prod_{1}^{l} \frac{i}{k_{a}^{2}-m_{a}^{2}+i \varepsilon} \prod_{J=1}^{n} \delta^{(D)}\left(p_{j}-\varepsilon_{j a} k_{a}\right)
$$

up to numerical constants. $D$ denotes the integral dimension of the pseudo-Euclidian space with signature $(+,(D-1)-), p_{j}$ denotes the sum of the external momenta ending at vertex $j$ and $\varepsilon_{j a}$ a topological matrix which depends on the orientation of the internal lines of the graph:

$\varepsilon_{j a}=+1$ if the line a points away from the vertex $j$

$\varepsilon_{j a}=-1$ if the line a points into the vertex $j$

and 0 otherwise. 
We introduce the parametric integral representation of the propagator

and

$$
\frac{i}{k^{2}-m^{2}+i \varepsilon}=\int_{0}^{\infty} d \alpha e^{i \alpha\left(k^{2}-m^{2}+i \varepsilon\right)}
$$

$$
\delta^{(D)}(k)=\frac{1}{(2 \pi)^{D}} \int_{-\infty}^{\infty} d^{D} x e^{i k x} .
$$

All scalar products are taken in the sense of the pseudo-Euclidian metric. After integration of the gaussian integrals over the $k$ and $x$ variables, we obtain the parametric integral representation of the Feynman amplitude:

$$
I=i^{L}(-i \pi)^{\frac{L D}{2}} \delta^{(D)}\left(\sum_{j} p_{J}\right) \int_{0}^{\infty} \cdots \int_{0}^{\infty} d \alpha_{1} \ldots d \alpha_{l} e^{-i \sum \alpha_{a}\left(m_{a}^{2}-i \varepsilon\right)} \frac{e^{i p_{i} d_{i j}^{1} p_{j}}}{P^{D / 2}}
$$

where $P$ is a homogeneous polynomial in the $\alpha$ 's; $d_{i j}^{-1}$ is obtained as follows: we define $\widetilde{d_{i J}}=\sum_{a=1}^{l} \frac{\varepsilon_{i a} \varepsilon_{j a}}{\alpha_{a}}$ as a $n \times n$ matrix; since $\sum_{i} \varepsilon_{i a}=0$, the determinant of $\tilde{d}$ is zero; $d_{i j}^{-1}$ is the inverse of a $(n-1) \times(n-1)$ diagonal minor of $\tilde{d}$.

Of course, the integral (II.4) is possibly divergent; we shall define its finite part in the following section.

The above representation can be found extensively in the literature [6].

Let us just mention some useful properties:

1) The polynomial $P$ is homogeneous in the $\alpha$ 's with degree $L$, the number of loops of the graph.

$P$ is positive when all $\alpha$ 's are positive; it vanishes as $\varrho^{L_{2}}$ when all $\alpha$ 's corresponding to a subdiagram with $L_{i}$ loops vanish as $\varrho$.

Each term in $P$ is a product of $L$ different $\alpha$ 's attached to $L$ lines which must be cut if we want to transform the graph into a tree-graph.

2) The matrix $d_{i j}^{-1}$ is the ratio of two polynomials $\frac{N_{i j}}{P}$ where $N_{i j}$ is a homogeneous polynomial in the $\alpha$ 's of degree $L+1 ; d_{i j}^{-1}$ has no singularities. There exist many other properties for which we refer to [6].

Let us define some concepts which will be used later on.

A subdiagram is defined by a family of $\alpha$ 's. A family of $\alpha$ 's defines a set of lines and vertices (all the end-points of these lines).

A family of $\alpha$ 's is said to be proper (or one-particle irreducible) if in the corresponding subdiagram each line belongs to at least one loop.

A family of $\alpha$ 's is connected if the corresponding subdiagram is connected.

A family of $\alpha$ 's is said to be a subgraph if any two vertices in the corresponding subdiagram are joined by all the lines which already 
joined them in the original graph. The concepts of union, intersection, inclusion and the symbols $\cup, \cap, C$ will be understood over sets of lines and vertices.

Two families of $\alpha$ 's are disjoint if their intersection is empty. If two families of $\alpha$ 's are neither disjoint nor contained one inside the other, the families are said to overlap.

A forest is a set of non overlapping families of $\alpha$ 's. This definition differs from Zimmermann's [5] who only considered forests of proper, connected, divergent subdiagrams, the so-called renormalization parts.

As mentioned before, the integral (II.4) may diverge due to the zeros of the polynomial $P$. Given a family of $\alpha$ 's with $L_{i}$ loops and $l_{i}$ lines, the integral diverges if $L_{i} D-2 l_{i} \geqq 0$. Such families of $\alpha$ 's are said to be divergent; $L_{i} D-2 l_{i}$ is the so-called superficial degree of divergence of the subdiagram.

\section{Renormalized Feynman Amplitude}

Definition. The renormalized Feynman amplitude is:

$$
\begin{gathered}
I_{R}=i^{L}(-i \pi)^{\frac{L D}{2}} \delta^{(D)}\left(\Sigma p_{j}\right) \int_{0}^{\infty} \ldots \int_{0}^{\infty} d \alpha_{1} \ldots d \alpha_{l} e^{-i \sum \alpha_{1}\left(m_{a}^{2}-i \varepsilon\right)} \\
\cdot \prod_{i}\left(1-\mathscr{T}_{\mathscr{P}_{i}}^{-2 l i}\right)\left\{\frac{e^{i p_{i} d_{i j}^{1} p_{J}}}{P^{D / 2}}\right\} .
\end{gathered}
$$

The operators $\mathscr{T}$ denote generalized Taylor operators: given a function $f(x)$ which behaves as $\frac{1}{x^{p}}$ (integer $p \gtrless 0$ ) at $x=0, \mathscr{T}^{n} f$ is equal to $\frac{1}{x^{p}}$ times the Taylor expansion of $g(x)=x^{p} f(x)$ up to order $n+p$. A complete definition and various properties are gathered in Appendix I.

The product $\prod_{i}$ runs over all possible families of $\alpha$ 's, that is $\left(2^{l}-1\right)$ families. For each family $\mathscr{S}_{i}$ with $l_{i}$ lines, $\mathscr{T}_{\mathscr{S}_{i}}{ }^{2 l_{i}}$ acts upon the dilatation variable $\varrho_{i}$ of the family when $\sqrt{\alpha_{i}}$ is dilated into $\varrho_{i} \sqrt{\alpha_{i}}$.

Let us define

$$
Z=\frac{e^{i p_{i} d_{i j}^{1} p_{j}}}{P^{D / 2}} .
$$

Although the $\mathscr{T}$ operators do not commute in general, they do commute when the corresponding families of $\alpha$ 's do not overlap; however it will be shown in Appendix II that the complete product of $(1-\mathscr{T})$ 's does not depend on their order. Consequently $\prod_{i}\left(1-\mathscr{T}_{\mathscr{S}_{i}}^{-2 l_{i}}\right) Z$ behaves for 
any family of $l_{j} \alpha$ 's as a pole of order less than $l_{J}$ and thus the integrability at the lower limit of integration is ensured.

This section is devoted to the proof of the following identity

$$
\prod_{i}\left(1-\mathscr{T}_{\mathscr{S}_{i}}^{-2 l_{2}}\right) Z=\left[1+\sum_{\substack{\text { non empty } \\ \text { forests }}} \Pi\left(-\mathscr{T}_{\overline{\mathscr{S}}_{i}}^{-2 l_{2}}\right)\right] Z
$$

where the sum runs over the set of all non-empty forests; this identity will be further reduced to a sum over the set of non-empty forests of proper, connected, divergent subgraphs.

In contradistinction to Appelquist's result [4], our proof is valid for graphs occurring in a non renormalizable theory.

\section{Theorem $1^{1}$.}

$$
\prod_{i}\left(1-\mathscr{T}_{\mathscr{S}_{i}}^{-2 l_{l}}\right) Z=\left[1+\sum_{\mathscr{F}} \prod_{\mathscr{S}_{j} \in \mathscr{F}}\left(-\mathscr{T}_{\mathscr{S}_{j}}^{-2 l_{j}}\right)\right] Z
$$

where the sum runs over the forests of families of $\alpha$ 's whether proper, connected, divergent or not.

Proof. Since $\prod_{i}\left(1-\mathscr{T}_{\mathscr{S}_{i}}^{-2 l_{i}}\right) Z$ is independent of the order of the $\mathscr{S}_{i}$ 's, we order it in such a way that the number of lines decreases from left to right. We then establish recursively the following identity:

$$
\prod_{i}\left(1-\mathscr{T}_{\mathscr{S}_{i}}^{-2 l_{i}}\right) Z=\prod_{i \geqq m}\left(1-\mathscr{T}_{\mathscr{S}_{i}}^{-2 l_{i}}\right)\left[1+\sum_{\mathscr{F} \in \mathscr{E}_{m-1}} \prod_{\mathscr{S}_{j} \in \mathscr{F}}\left(-\mathscr{T}_{\mathscr{S}_{j}}\right)\right] Z
$$

where $\mathscr{E}_{m-1}$ is the set of forests built with elements at the right of $\mathscr{S}_{m-1}$ and eventually $\mathscr{S}_{m-1}$ itself.

The identity (III.5) is trivially verified for the nearest element to $Z$ and we shall prove it for order $m$.

Let us define $\mathscr{E}_{m-1}^{\prime} \subset \mathscr{E}_{m-1}$ the set of forests which contains at least one family of $\alpha$ 's overlapping with $\mathscr{S}_{m}$. Then the recursion proof amounts to the validity of the following identity:

$$
\prod_{i \geqq m+1}\left(1-\mathscr{T}_{\mathscr{S}_{l}}\right)\left(-\mathscr{T}_{\mathscr{S}_{m}}\right)\left[\sum_{\mathscr{F} \in \mathscr{\sigma}_{m}^{\prime}-1} \prod_{\mathscr{S}_{j} \in \mathscr{F}}\left(-\mathscr{T}_{\mathscr{S}_{j}}\right)\right] Z=0 .
$$

The general idea for the proof of (III.6) is the following: given two overlapping families of $\alpha$ 's $\mathscr{S}_{1}$ and $\mathscr{S}_{2}$, we observe from the properties of the $\mathscr{T}$ 's and $(1-\mathscr{T})$ 's and from the topological relation

$$
l_{\cup}=l_{1}+l_{2}-l_{\cap},
$$

that

$$
\left(-\mathscr{T}_{\mathscr{S}_{1}}^{-2 l_{1}}\right)\left(1-\mathscr{T}_{\mathscr{S}_{1} \cup \mathscr{S}_{2}}^{-2 l_{\breve{S}}}\right)\left(1-\mathscr{T}_{\mathscr{S}_{1} \cap \mathscr{S}_{2}}^{-2 l_{n}}\right)\left(-\mathscr{T}_{\mathscr{S}_{2}}^{-2 l_{2}}\right) Z=0 .
$$

${ }^{1}$ It is interesting to remark that this theorem holds for any function $Z$ such that the product of $(1-\mathscr{T})$ 's is independent of their order. 
The problem here is to generalize this property to a complete forest. This highly technical part of the proof is given in Appendix III. On the right hand side of (III.4), each term of the sum is now a commutative product of $\mathscr{T}$ 's since the corresponding families of $\alpha$ 's do not overlap.

Theorem 2.

$$
\Pi\left(1-\mathscr{T}_{\mathscr{S}_{i}}^{-2 l_{\imath}}\right) Z=\left[1+\sum_{\mathscr{F}} \prod_{\mathscr{S}_{j} \in \mathscr{F}}\left(-\mathscr{T}_{\mathscr{S}_{j}}^{-2 l_{j}}\right)\right] Z
$$

where the sum runs over the forests of divergent families of $\alpha$ 's, whether proper, connected or not.

Proof. Let us consider a term in the right hand side of (III.4) that contains a convergent family of $\alpha$ 's. From the commutation property of the $\mathscr{T}$ 's, we can bring the corresponding $\mathscr{T}$ to the right, next to $Z$. $Z$ has a pole of order $L_{i} D$ for this family and since $-2 l_{i}+L_{i} D$ is negative, $-\mathscr{T}_{\mathscr{S}_{i}}^{-2 l_{i}} Z$ vanishes [Appendix (A.I-5)]. In particular note that treegraphs are eliminated at this stage.

From now on, we shall feel free, if needed, to reintroduce in the sum (III.9) any product of commuting $\mathscr{T}$ 's containing convergent families of $\alpha$ 's.

Theorem 3.

$$
\Pi\left(1-\mathscr{T}_{\mathscr{S}_{\imath}}^{-2 l_{i}}\right) Z=\left[1+\sum_{\mathscr{F}} \prod_{\mathscr{S}_{j} \in \mathscr{F}}\left(-\mathscr{T}_{\mathscr{S}_{j}}^{-2 l_{j}}\right)\right] Z
$$

where the sum runs over the forests of proper, divergent families of $\alpha$ 's, whether connected or not.

Proof. Let us consider a term in the right hand side of (III.9) that contains an improper divergent family of $\alpha$ 's $\mathscr{S}_{1}$. This term might also contain other improper divergent families of $\alpha$ 's contained in $\mathscr{S}_{1}$. We pick among them $\mathscr{S}_{1}^{\prime}$ which does not contain any other improper divergent family. Then, the maximal proper divergent family of $\alpha$ 's $\mathscr{S}_{2}$ contained in $\mathscr{S}_{1}^{\prime}$ forms a forest with the other elements of the considered term. We can group the terms

$$
\ldots\left(1-\mathscr{T}_{\mathscr{\mathscr { S }}_{2}}^{-2 l_{2}}\right)\left(-\mathscr{T}_{\mathscr{S}_{1}^{\prime}}^{-2 l_{1}^{\prime}}\right) Z
$$

where the corresponding $\mathscr{T}$ operators are written to the right, next to $Z$. After dilatation of the $\sqrt{\alpha}$ 's belonging to $\mathscr{S}_{1}^{\prime}$ by $\varrho$ and to $\mathscr{S}_{2}$ by $\mu, Z(\varrho, \varrho \mu)$ has the form $\frac{1}{(\varrho \mu)^{L D}} g(\varrho, \varrho \mu)$ where $L$ denotes the common number of loops of $\mathscr{S}_{1}^{\prime}$ and $\mathscr{S}_{2}$ and $g$ has a Taylor expansion in $\varrho$ and $\varrho \mu$. Then, we can write:

$$
\left(-\mathscr{T}_{\mathscr{S}_{1}^{i}}^{2 l_{1}^{\prime}}\right) Z=\frac{1}{(\varrho \mu)^{L D}} \sum_{\substack{k_{i} \geqq 0 \\ k_{1}+k_{2} \leqq-2 l_{1}^{\prime}+L D}} \frac{\varrho^{k_{1}}}{k_{1} !} \frac{(\varrho \mu)^{k_{2}}}{k_{2} !} g^{\left(k_{1}\right),\left(k_{2}\right)}(0,0) .
$$


Clearly, it behaves in $\mu$ as $\mu^{q}$ with $q \leqq-2 l_{1}^{\prime}$. Since $l_{2}<l_{1}^{\prime},\left(1-\mathscr{T}_{\mathscr{S}_{2}}^{-2 l_{2}}\right)$ $\cdot\left(-\mathscr{T}_{\mathscr{Y}_{1}^{\prime}}^{2}{ }^{2 l_{1}}\right) Z$ vanishes.

Theorem 4.

$$
\Pi\left(1-\mathscr{T}_{\mathscr{S}_{i}}^{-2 l_{\imath}}\right) Z=\left[1+\sum_{\mathscr{F}} \prod_{\mathscr{S}_{j} \in \mathscr{F}}\left(-\mathscr{T}_{\mathscr{S}_{j}}^{-2 l_{j}}\right)\right] Z
$$

where the sum runs over the forests of proper, connected, divergent families of $\alpha$ 's.

Proof. Let us consider a term in the right hand side of (III.10) that contains a disconnected, proper, divergent family of $\alpha$ 's $\mathscr{S}=\mathscr{S}_{1} \cup \cdots \cup \mathscr{S}_{k}$ where the $\mathscr{S}_{i}$ 's are mutually disjoint.

It is always possible to gather the following terms:

$$
\ldots\left(-\mathscr{T}_{\mathscr{S}}^{-2 l}\right)\left(1-\mathscr{T}_{\mathscr{S}_{1}}^{-2 l_{1}}\right) \ldots\left(1-\mathscr{T}_{\mathscr{S}_{k}}^{-2 l_{k}}\right) Z
$$

where again, the corresponding $\mathscr{T}$ 's are written to the right, next to $Z$. Using the same argument as in Theorem 3, it is easy to check that after dilatation of the $\sqrt{\alpha}$ 's belonging to $\mathscr{S}$ by $\varrho$ and to $\mathscr{S}_{i}$ by $\mu_{i}$, $\prod_{1}^{k}\left(1-\mathscr{T}_{\mathscr{S}_{i}}^{-2 l_{i}}\right) Z\left(\varrho \mu_{i}\right)$ behaves in $\varrho$ as a power greater than $2 \sum_{1}^{k}\left(-l_{i}\right)=-2 l$ so that (III.14) vanishes.

This achieves the proof that the product of $(1-\mathscr{T})$ 's over all families of $\alpha$ 's is equivalent to the sum over Zimmermann's forests. At this stage, we still have to eliminate the families of $\alpha$ 's which are not subgraphs.

Theorem 5.

$$
\Pi\left(1-\mathscr{T}_{\mathscr{\mathscr { S }}_{\imath}}^{-2 l_{l}}\right) Z=\left[1+\sum_{\mathscr{F}} \prod_{\mathscr{S}_{J} \in \mathscr{F}}\left(-\mathscr{T}_{\mathscr{S}_{J}}^{-2 l_{J}}\right)\right] Z
$$

where the sum runs over the forests of proper, connected, divergent subgraphs.

Proof. Let us consider a term in the right hand side of (III.13) that contains a family of $\alpha$ 's $\mathscr{S}_{1}$ which is not a subgraph, i.e. it is necessary to add further lines but no vertices to obtain a subgraph. Let us add one such line to $\mathscr{S}_{1}$ to get the family $\mathscr{S}_{2} . \mathscr{S}_{2}$ has one line and one loop more than $\mathscr{I}_{1}$. Again, we gather the following terms

$$
\ldots\left(1-\mathscr{T}_{\mathscr{S}_{2}}^{-2 l_{2}}\right)\left(-\mathscr{T}_{\mathscr{S}_{1}}^{-2 l_{1}}\right) Z
$$

where the $\mathscr{T}$ 's are written close to $Z$.

After dilatation of the $\sqrt{\alpha}$ 's belonging to $\mathscr{S}_{1}$ by $\varrho$ and to $\mathscr{S}_{2}$ by $\mu$,

$$
P(\varrho \mu, \mu)=\varrho^{2 L} \mu^{2(L+1)} P^{\prime}(\varrho \mu, \varrho)
$$

as can easily be seen from the fact that there is only one $\alpha$ dilated by $\mu^{2}$ alone. 
The same property holds for the numerator of $d_{i j}^{-1}$. Then, similar considerations as in Theorem 3 and 4 show that (III.16) vanishes.

By repeated application of this property, adding each time one more line and no vertices to $\mathscr{S}_{1}$, we arrive at the complete subgraph which is the only contribution to the right hand side of (III.15).

Now,

$$
\begin{gathered}
I_{R}=i^{L}(-i \pi)^{\frac{L D}{2}} \delta^{(D)}\left(\Sigma p_{j}\right) \int_{0}^{\infty} \ldots \int_{0}^{\infty} d \alpha_{1} \ldots d \alpha_{l} \\
\cdot e^{-i \sum \alpha_{a}\left(m_{a}^{2}-i \varepsilon\right)}\left[1+\sum_{\mathscr{F}} \prod_{\mathscr{S}_{i} \in \mathscr{F}}\left(-\mathscr{T}_{\mathscr{S}_{i}}^{-2 l_{l}}\right)\right]\left\{\frac{e^{i p_{i} d_{\overline{i j}}^{1} p_{j}}}{P^{D / 2}}\right\}
\end{gathered}
$$

where the sum runs over all non empty forests of proper, connected divergent subgraphs.

Note. We have proved theorems similar to Theorems $2-5$ for the product of $(1-\mathscr{T})$ 's operating upon all families of $\alpha$ 's; namely we have shown that it is equivalent to the product of $(1-\mathscr{T})$ 's over all proper, connected, divergent subgraphs and that this product is independent of the order of the operators $(1-\mathscr{T})$. [This proof makes extensive use of identity (A.I-13).]

From a purely computational point of view, it is often easier to calculate $\Pi(1-\mathscr{T})$ than to perform the sometimes tedious decomposition into forests. At this point, it is worthwhile to emphasize an important property of the complete product $\Pi(1-\mathscr{T})$ in (III.1): this product seems to depend on the structure of the graph, since we have to extract the pole in each family before performing the Taylor expansion. However, we always know an upper bound $M$ on the order of the pole which might occur in any family of $\alpha$ 's after a certain number of $(1-\mathscr{T})$ 's have been applied. Then:

$$
R_{l} Z \equiv \prod_{i=1}^{2^{l}-1}\left(1-\mathscr{T}_{\mathscr{S}_{i}}^{-2 l_{i}}\right) Z=\prod_{i=1}^{2^{l}-1}\left(1-T_{\varrho_{i}}^{M-2 l_{l}}\right)\left\{\prod_{j=1}^{2^{l}-1} \varrho_{j}^{M} \tilde{Z}\right\}_{\varrho_{l}=1}
$$

where $\tilde{Z}$ is obtained from $Z$ by dilatation of all $\sqrt{\alpha_{j}} \in \mathscr{S}_{i}$ by $\varrho_{i}$. Since the product in (III.19) runs over all possible families $\mathscr{S}_{i}$, we do obtain a substraction operator completely independent of the topology of the graph (except for the total number of internal lines).

\section{Finite Part of a Feynman Amplitude in Momentum Space}

In this section, our purpose is to show that the finite part of a Feynman amplitude as defined by (III.18) is equal to the finite part as defined by Zimmermann [5] in momentum space. 
Given the Feynman amplitude $I\left(p_{1}, \ldots, p_{n-1}\right)$ associated to a Feynman graph, the finite part is given by Bogoliubov's recursive $R$-operation: the integrand in $p$-space $I_{\Gamma}$ has to be replaced by

$$
R_{\Gamma}=\left(1-T_{\Gamma}^{d(\Gamma)}\right) \bar{R}_{\Gamma}
$$

where, for any proper, connected, divergent subdiagrams (renormalization parts) $\gamma$

$$
\begin{gathered}
\bar{R}_{\gamma}=I_{\gamma}+\sum_{\left\{\gamma_{1}, \ldots, \gamma_{c}\right\}} I_{\frac{\gamma}{\gamma_{1} \ldots \gamma_{c}}} \prod_{j=1}^{c} \mathcal{O}_{\gamma_{j}}, \\
\mathcal{O}_{\gamma}=-T_{\gamma}^{d(\gamma)} \bar{R}_{\gamma} .
\end{gathered}
$$

In these formulae, $\gamma_{1}, \ldots \gamma_{c}$ are mutually disjoint renormalization parts of $\gamma(\gamma$ excluded $), \frac{\gamma}{\gamma_{1} \ldots \gamma_{c}}$ denotes the reduced $\gamma$ subgraph where $\gamma_{1}, \ldots, \gamma_{c}$ are contracted to points and $T_{\gamma}^{d(\gamma)}$ is the Taylor expansion over the $n_{\gamma}-1$ external momenta of $\gamma$, at zero momentum up to the superficial degree of divergence

$$
d(\gamma)=D L-2 l .
$$

( $L$ and $l$ are the number of loops and of lines of $\gamma$ ).

The solution to the recursive operation (IV.1.2.3) was proved by Zimmermann to be

$$
R_{\Gamma}=\left[1+\sum_{\text {forests } \mathscr{F}} \prod_{\gamma \in \mathscr{F}}\left(-T_{\gamma}^{d(\gamma)}\right)\right] I_{\Gamma}
$$

where the Taylor operators are ordered in such a way that $T_{\gamma_{1}}$ stands to the left of $T_{\gamma_{2}}$ if $\gamma_{2} \subset \gamma_{1}$, and $\mathscr{F}$ are the forests of renormalization parts.

Note. If $\Gamma$ has no overlapping divergencies, the above formula is equivalent to

$$
R_{\Gamma}=\Pi\left(1-T_{\gamma}^{d(\gamma)}\right) I_{\Gamma},
$$

where the ordered product is taken over all renormalization parts of $\Gamma$.

In order to calculate $\tilde{I}_{\gamma}$ :

$$
\begin{aligned}
\tilde{I}_{\gamma} \delta^{(D)}\left(\sum_{1}^{n} q_{j}\right)= & \int \prod_{a=1}^{l} d^{D} k_{a}\left(-T_{\gamma}^{d(\gamma)}\right) \prod_{a=1}^{l} \frac{i}{k_{a}^{2}-m_{a}^{2}+i \varepsilon} \\
& \cdot \prod_{j=1}^{n-1} \delta^{(D)}\left(q_{j}-\varepsilon_{j a} k_{a}\right) \delta^{(D)}\left(\sum_{1}^{n} q_{j}\right)
\end{aligned}
$$

the integrand $I_{\gamma}$ is generally expressed as a function of loop variables and external momenta $q$ by integration of the $n-1 \delta^{(D)}$ distributions. Then, 
the Taylor substraction operator acts upon those propagators, the momenta of which are functions of the $q$ 's.

However, since the propagators as expressed in (IV.7) are independent of the external legs, we can perform the Taylor substractions over the $(n-1) \delta^{(D)}$ distributions; by suitable integration by parts we recover the preceding results. Moreover, this second method is convenient for the parametric integral representation. Indeed, after dilatation of the external momenta by $\varrho$, it leads to

$$
-\left.T_{\varrho}^{d(\gamma)} \prod_{j=1}^{n-1} \delta^{(\boldsymbol{D})}\left(\varrho q_{j}-\varepsilon_{j a} k_{a}\right)\right|_{\varrho=1}
$$

so that $\tilde{I}_{\gamma}$ is simply

$$
\begin{gathered}
\tilde{I}_{\gamma} \delta^{(D)}\left(\sum_{1}^{n} q_{j}\right)=\int \prod_{a=1}^{l} d^{D} k_{a} \prod_{a=1}^{l} \frac{i}{k_{a}^{2}-m_{a}^{2}+i \varepsilon} \\
\cdot\left[\left(-T_{\varrho}^{d(\gamma)}\right)\left\{\frac{1}{\varrho^{(n-1) D}} \prod_{j=1}^{n} \delta^{(D)}\left(q_{J}-\varepsilon_{j a} \frac{k_{a}}{\varrho}\right)\right\}\right]_{\varrho=1} .
\end{gathered}
$$

It is important to note that, in (IV.9), a $\frac{1}{\varrho}$ factor is now attached to each internal momentum inside the $\delta^{(D)}$ distribution. This mechanism and the resulting property can be generalized to a product of several $T_{\gamma_{\mathrm{t}}}$ operators relative to several subdiagrams $\gamma_{i}$ as long as the subdiagrams form a forest and $T_{\gamma_{i}}$ stands to the left of $T_{\gamma_{j}}$ if $\gamma_{j} \subset \gamma_{i}$. We thus obtain for each forest $\mathscr{F}$.

$$
\begin{gathered}
\int \prod_{a=1}^{l} d^{D} k_{a} \prod_{a=1}^{l} \frac{i}{k_{a}^{2}-m_{a}^{2}+i \varepsilon} \\
\cdot\left[\prod_{\gamma_{i} \in \mathscr{F}}\left(-T_{\varrho_{i}}^{d\left(\gamma_{i}\right)}\right)\left\{\prod_{i} \frac{1}{\varrho_{i}^{\left(\eta_{\gamma_{i}}-1\right) D}} \prod_{j=1}^{n} \delta^{(D)}\left(p_{j}-\sum_{a=1}^{l} \varepsilon_{j a} \frac{k_{a}}{\prod_{k_{a} \in \gamma_{l}} \varrho_{l}}\right)\right\}\right]_{\varrho_{i}=1}
\end{gathered}
$$

We now introduce for Eq. (IV.5) the parametric integral representation as in Section II; after integration over the $k$ momenta and use of (IV.10), a factor $\varrho_{i}^{2}$ remains attached to all $\alpha$ 's which belong to $\gamma_{i}$.

The finite part for the Feynman amplitude as defined by Zimmermann is now found to be

$$
\begin{gathered}
I_{R}=i^{L}(-i \pi)^{\frac{L D}{2}} \delta^{(D)}\left(\sum_{j} p_{j}\right) \int_{0}^{\infty} \cdots \int_{0}^{\infty} d \alpha_{1} \ldots d \alpha_{l} e^{-i \sum \alpha_{a}\left(m_{a}^{2}-i \varepsilon\right)} \\
\cdot\left[\left[1+\sum_{\mathscr{F}} \prod_{\gamma_{i} \in \mathscr{F}}\left(-T_{\varrho_{i}}^{d\left(\gamma_{i}\right)}\right)\right]\left\{\prod_{i} \varrho_{i}^{L_{i} D} \frac{e^{i p_{1} d_{i j}^{1}{ }^{1}\left(\varrho_{k}^{2}\right) p_{j}}}{P\left(\varrho_{k}^{2}\right)^{D / 2}}\right\}\right]_{\varrho_{i}=1}
\end{gathered}
$$


From the definition of the $\mathscr{T}$ operators (A.I-3), we finally obtain Eq. (III.13). The equivalence between Zimmermann's definition of the finite part and ours is thus established.

\section{Generalization to Theories with Spin and Derivative Couplings}

The Feynman amplitude for graphs arising in such theories can be written

$$
I=\int \prod_{a=1}^{l} d^{D} k_{a} \prod_{a=1}^{l} \frac{i Q_{a}\left(k_{a}^{\mu}\right)}{k_{a}^{2}-m_{a}^{2}+i \varepsilon} \prod_{j=1}^{n}\left\{\mathscr{P}_{j}\left(p_{J}, k_{a}^{\mu}\right) \delta^{(D)}\left(p_{j}-\varepsilon_{j a} k_{a}\right)\right\}(\mathrm{V} .1)
$$

up to numerical constants and $\gamma$ matrices. The polynomials $Q_{a}$ of degree $d_{a}$ are characteristic of the spin of the particles and the polynomials $\mathscr{P}_{j}$ of degree $d_{j}$ are homogeneous in the internal and external momenta ending at vertex $j$.

We use the integral representation of the propagator

$$
\frac{i Q\left(k^{\mu}\right)}{k^{2}-m^{2}+i \varepsilon}=\left.\int_{0}^{\infty} d \alpha Q\left(\frac{1}{i \sqrt{\alpha}} \frac{\partial}{\partial z_{\mu}}\right) e^{i \alpha\left(k^{2}+\frac{k \cdot z}{\sqrt{\alpha}}-m^{2}+i \varepsilon\right)}\right|_{z=0}
$$

where the factor $\alpha^{1 / 2}$ is introduced for convenience.

The parametric integral representation for the Feynman amplitude (V.1) is found to be

$$
\begin{aligned}
I=i^{L}(-i \pi)^{\frac{L D}{2}} \delta^{(D)}\left(\Sigma p_{J}\right) & \int_{0}^{\infty} \cdots \int_{0}^{\infty} d \alpha_{1} \ldots d \alpha_{l} e^{-i \sum \alpha_{a}\left(m_{a}^{2}-i \varepsilon\right)} \\
& \cdot\left\{\prod_{a=1}^{l} Q_{a}\left(\frac{1}{i \sqrt{\alpha_{a}}} \frac{\partial}{\partial z_{a}^{\mu}}\right) \prod_{j=1}^{n} \mathscr{P}_{j}\left(p_{J}, \frac{1}{i \sqrt{\alpha_{a}}} \frac{\partial}{\partial z_{a}^{\mu}}\right) Z\right\}_{z_{a}=0}
\end{aligned}
$$

with

$$
\begin{aligned}
Z= & \frac{1}{P^{D / 2}} \exp i\left[p_{i} d_{i j}^{-1} p_{J}+p_{i} d_{i j}^{-1} \varepsilon_{j a} \frac{z_{a}}{\sqrt{\alpha_{a}}}\right. \\
& \left.+\frac{1}{4} \frac{z_{a}}{\sqrt{\alpha_{a}}}\left(\varepsilon_{i a} d_{i j}^{-1} \varepsilon_{j b}-\alpha_{a} \delta_{a b}\right) \frac{z_{b}}{\sqrt{\alpha_{b}}}\right] \\
= & Z(\alpha, p, z) .
\end{aligned}
$$

It can be shown [6] that $d_{i j}^{-1} \varepsilon_{j a}$ is proportional to $\alpha_{a}$ and $\varepsilon_{i a} d_{i j}^{-1} \varepsilon_{j b}-\alpha_{a} \delta_{a b}$ is proportional to $\alpha_{a} \alpha_{b}$. Then, $d_{i j}^{-1} \frac{\varepsilon_{j a}}{\sqrt{\alpha_{a}}}$ is the ratio of two polynomials 
in $\sqrt{\alpha}$ 's: $\frac{N_{i a}}{P}$ where $N_{i a}$ is homogeneous of degree $2 L+1$ in the $\sqrt{\alpha}$ 's; $\frac{N_{i a}}{P}$ and $\tilde{k}_{a}=p_{i} d_{i j}^{-1} \frac{\varepsilon_{j a}}{\alpha_{a}}$ have no singularities. Also, $\frac{\left(\varepsilon_{i a} d_{i j}^{-1} \varepsilon_{j b}-\alpha_{a} \delta_{a b}\right)}{\sqrt{\alpha_{a} \alpha_{b}}}$ is the ratio of two polynomials in $\sqrt{\alpha}$ 's: $\frac{N_{a b}}{P}$ where $N_{a b}$ is homogeneous of degree $2 L$ in the $\sqrt{\alpha}$ 's; $\frac{N_{a b}}{P}$ has no singularities but

$$
\Delta_{a b}=\frac{1}{2 i \sqrt{\alpha_{a} \alpha_{b}}} \frac{N_{a b}}{P}
$$

is singular as a pole of degree 2 in the $\sqrt{\alpha}$ 's for any proper connected family ${ }^{2}$ of $\alpha$ 's containing both $\alpha_{a}$ and $\alpha_{b}\left(\Delta_{a a}\right.$ is singular for any proper connected family of $\alpha$ 's containing $\alpha_{a}$ ).

It will be useful to introduce the following notation

$$
\prod_{a=1}^{l} Q_{a}\left(k_{a}\right) \prod_{j=1}^{n} \mathscr{P}_{j}\left(p_{j}, k_{a}\right)=\sum_{\sigma} \prod_{j=1}^{\lambda} p_{j} \prod_{a=1}^{v} k_{a}
$$

where we omit Lorentz indices and constant coefficients:

Given a diagram $\mathscr{S}_{i}$, each term in (V.5) is of degree $\delta_{i}(\sigma) \leqq \sum_{1}^{l_{2}} d_{a}+\sum_{1}^{n_{i}} d_{j}$ in the momenta $k_{a}$ internal to $\mathscr{S}_{i}$. We define the superficial degree of divergence of $\mathscr{S}_{i}$ for a given term in (V.5) as:

$$
d\left(\mathscr{S}_{i}, \sigma\right)=D L_{i}-2 l_{i}+\delta_{i}(\sigma)
$$

Note that this definition differs from Zimmermann's definition [5]

$$
d\left(\mathscr{S}_{i}\right)=D L_{i}-2 l_{i}+\sum_{1}^{l_{i}} d_{a}+\sum_{1}^{n_{j}} d_{j}
$$

for all terms in (V.5). For the sake of completeness, we can perform the scalar decomposition of the integral (V.3) using ${ }^{3}$

$$
\begin{aligned}
& \qquad\left\{\prod_{1}^{v}\left(\frac{1}{i \sqrt{\alpha_{a}}} \frac{\partial}{\partial z_{a}^{\mu}}\right) Z(\alpha, p, z)\right\}_{z_{a}=0} \\
& =\sum_{k=0}^{E\left(\frac{v}{2}\right)}\left[g^{\mu_{1} \mu_{2}} \Delta_{a_{1} a_{2}} \ldots g^{\mu_{2 k-1 \mu_{2 k}}} \Delta_{a_{2 k-1} a_{2 k}} \cdot k_{a_{2 k+1}}^{\mu_{2 k+1}} \ldots k_{a_{v}}^{\mu_{\nu}}+\operatorname{sym} .\right] Z(\alpha, p, 0) \\
& \frac{{ }^{2} \text { For improper or non connected families, only the maximal proper connected }}{\text { families contained into it, contribute to the pole. }} \\
& { }^{3} E(*) \text { denotes the integral part of } x .
\end{aligned}
$$


and obtain, up to constant coefficients:

$$
\left.\begin{array}{rl}
I=\sum_{\sigma}\left\{( \prod _ { j = 1 } ^ { \lambda } p _ { j } ^ { \mu _ { j } } ) ^ { E } \sum _ { \substack { k = 0 \\
2 } } ^ { E ( \frac { v } { 2 } ) } \left[g^{\mu_{1} \mu_{2}} \ldots g^{\mu_{2 k} \mu_{2 k-1}} p_{r_{1}}^{\mu_{2 k+1}} \ldots\right.\right. \\
\left.\ldots p_{r_{\nu-2 k}}^{\mu_{\nu}} I_{\left(a_{1} \ldots a_{2 k}\right)\left(a_{2 k+1} \ldots a_{v}\right)}^{r_{1} \ldots r_{\nu-2 k}}+\text { sym. } .\right]
\end{array}\right\}
$$

where we sum over $r_{i}$ from 1 to $n-1$ and where the scalar integrals are

$$
\begin{aligned}
& I_{\left(a_{1} \ldots a_{2 k}\right)\left(a_{2 k+1} \ldots a_{v}\right)}^{r_{1} \ldots r_{\nu-2 k}}=i^{L}(-i \pi)^{\frac{L D}{2}} \delta^{(D)}\left(\Sigma p_{J}\right) \int_{0}^{\infty} d \alpha_{1} \ldots d \alpha_{l} e^{-i \sum_{1}^{L} \alpha_{a}\left(m_{a}^{2}-i \varepsilon\right)} \\
& \cdot\left[\Delta_{a_{1} a_{2}} \ldots \Delta_{a_{2 k-1} a_{2 k}}\left(d_{r_{1 J}}^{-1} \frac{\varepsilon_{j a_{2 k+1}}}{\alpha_{a_{2 k+1}}}\right) \ldots\left(d_{r_{v-2 k J}}^{-1} \frac{\varepsilon_{j a_{v}}}{\alpha_{a_{v}}}\right)\right] \frac{e^{i p_{2} d_{i j}{ }^{1} p_{j}}}{P^{D / 2}} .
\end{aligned}
$$

In Eqs. (V.8) and (V.9), "Sym" indicates that symmetrization on $\left(\begin{array}{l}\mu \\ a\end{array}\right)$ indices must be performed.

For a given term in the sum (V.5), we define the convergent families of $\alpha$ 's as the families such that $d\left(\mathscr{S}_{i}, \sigma\right)<0$.

Each integral $I_{(a)}^{(r)}$ in (V.10) has a pole for a given family of $\alpha$ 's of order at most equal to $L_{i} D+2 \beta_{i}$ where $\beta_{i}$ is the number of $\Delta_{a b}$ in (V.10) such that $\alpha_{a}$ and $\alpha_{b}$ belong to a maximal proper connected part of the family, $\left(\beta_{i} \leqq \frac{\delta_{i}^{a}(\sigma)}{2}\right)$. For convergent families of $\alpha$ 's, $d\left(\mathscr{S}_{i}, \sigma\right)<0$ implies $L_{i} D-2 l_{i}+2 \beta_{i}<0$; the converse is not true: it means that for any convergent family, the corresponding singularity in (V.10) is indeed integrable.

Definition. The renormalized Feynman amplitude is:

$$
\begin{aligned}
I_{R}= & i^{L}(-i \pi)^{\frac{L D}{2}} \delta^{(D)}\left(\Sigma p_{j}\right) \int d \alpha_{1} \ldots d \alpha_{l} e^{-i \sum \alpha_{a}\left(m_{a}^{2}-i \varepsilon\right)} \prod_{i}\left(1-\mathscr{T}_{\mathscr{S}_{i}}^{-2 l_{l}}\right) \\
& \cdot\left\{\prod_{a=1}^{l} Q_{a}\left(\frac{1}{i \sqrt{\alpha_{a}}} \frac{\partial}{\partial z_{a}}\right) \prod_{j=1}^{n} \mathscr{P}_{j}\left(p_{j}, \frac{1}{i \sqrt{\alpha_{a}}} \frac{\partial}{\partial z_{a}}\right) Z(\alpha, p, z)\right\}_{z_{a}=0} .
\end{aligned}
$$

Note that this definition is equivalent to

$$
\begin{gathered}
I_{R}=i^{L}(-i \pi)^{\frac{L D}{2}} \delta^{(D)}\left(\Sigma p_{J}\right) \int d \alpha_{1} \ldots d \alpha_{l} e^{-i \sum \alpha_{a}\left(m_{a}^{2}-i \varepsilon\right)} \sum_{\sigma} \prod_{j=1}^{\lambda} p_{j} \\
\cdot\left\{\prod_{a=1}^{v}\left(\frac{1}{i \sqrt{\alpha_{a}}} \frac{\partial}{\partial z_{a}}\right) \prod_{i}\left(1-\mathscr{T}_{\mathscr{S}_{l}}^{-2 l_{l}+\delta_{l}(\sigma)}\right) Z(\alpha, p, z)\right\}_{z=0}
\end{gathered}
$$

because $Z$ is regular in $z$.

The product $\prod_{i}$ runs over all possible families of $\alpha$ 's. 
In Appendix II, it is shown that the complete product of $(1-\mathscr{T})$ 's does not depend on their order. Consequently, the integrability at the lower limit of integration is ensured.

In Eq. (V.11), we can replace $\prod_{i}\left(1-\mathscr{T}_{\mathscr{S}_{i}}^{-2 l_{i}}\right)$ by $\left[1+\sum_{\text {forests }} \Pi\left(-\mathscr{T}_{\mathscr{S}_{i}}^{-2 l_{i}}\right)\right]$ as can be shown by simply repeating the Theorems 1 to 5 of Section II. The same property holds for Eq. (V.12).

In Theorem II, we eliminate the convergent families of $\alpha$ 's as defined before and consequently, the decomposition into forests is different for each term of the sum (V.5). Moreover, for a given term, some forests may contribute nothing, when applied to some integrands (V.10).

On the other hand, Zimmermann's formalism leads to Eq. (IV.5) where $T_{\gamma}^{d(\gamma)}$ operates over the external legs of $\gamma$ at zero momentum with a degree $d(\gamma)$ given by (V.7).

In contradistinction to Zimmermann's finite part, we define the minimal finite part of a Feynman amplitude as the amplitude obtained when performing the minimal number of substractions on each term of the sum (V.5). In the general case, the minimal and Zimmermann's power countings are inequivalent. However, due to the homogeneity of the polynomials $\mathscr{P}_{J}$ in the internal and external momenta, derivative couplings do not contribute to this difference.

Anyhow, both theories are related by a finite renormalization which can be calculated order by order.

Then, Eq. (IV.7) is generalized to:

$$
\begin{aligned}
\tilde{I}_{\gamma} \delta^{(D)}\left(\Sigma q_{j}\right)= & \sum_{\sigma} \prod_{j=1}^{\lambda} q_{J} \int \prod_{a=1}^{l} d^{D} k_{a}\left(-T_{\gamma}^{d(\gamma, \sigma)}\right) \prod_{a=1}^{l} \frac{i}{k_{a}^{2}-m_{a}^{2}+i \varepsilon} \\
& \cdot \prod_{a=1}^{v} k_{a} \prod_{j=1}^{n-1} \delta^{(D)}\left(q_{j}-\varepsilon_{j a} k_{a}\right) \delta^{(D)}\left(\Sigma q_{j}\right)
\end{aligned}
$$

with $d(\gamma, \sigma)$ defined in (V.6).

Here again, $T_{\gamma}$ operates over the $\delta$ distributions and, provided that the product of Taylor operators within each forest in (IV.5) is performed from minimal to maximal elements, the parametric integral representation for the renormalized amplitude can be calculated as in the scalar case; we obtain:

$$
\begin{aligned}
I_{R}= & i^{L}(-i \pi)^{\frac{L D}{2}} \delta^{(D)}\left(\Sigma p_{J}\right) \int d \alpha_{1} \ldots d \alpha_{l} e^{-i \sum \alpha_{a}\left(m_{a}^{2}-i \varepsilon\right)} \sum_{\sigma} \prod_{j=1}^{\lambda} p_{j} \\
& \cdot\left[\prod_{a=1}^{v}\left(\frac{1}{i \sqrt{\alpha_{a}}} \frac{\partial}{\partial z_{a}^{\mu}}\right)\left[1+\sum_{\mathscr{F}} \prod_{\gamma_{l} \in \mathscr{F}}\left(-T_{Q_{i}}^{d\left(\gamma_{i}, \sigma\right)}\right)\right]\right. \\
& \left.\cdot\left\{\prod_{i} \varrho_{i}^{L_{i} D} Z\left(\alpha \Pi \varrho_{l}^{2}, p, z\right)\right\}\right]_{\varrho_{l}=1, z_{a}=0}
\end{aligned}
$$


where the sum over the forests depends on the term considered in the sum over $\sigma$.

After introduction of the operators $\mathscr{T},(\mathrm{V} .14)$ is equivalent to

$$
\begin{aligned}
& I_{R}=i^{L}(-i \pi)^{\frac{L D}{2}} \delta^{(D)}\left(\sum p_{J}\right) \int d \alpha_{1} \ldots d \alpha_{l} e^{-i \sum \alpha_{a}\left(m_{a}^{2}-i \varepsilon\right)} \\
& \cdot \sum_{\sigma} \prod_{j=1}^{\lambda} p_{j}\left[\prod_{a=1}^{v}\left(\frac{1}{i \sqrt{\alpha_{a}}} \frac{\partial}{\partial z_{a}}\right)\left[1+\sum_{\mathscr{F}} \prod_{\mathscr{S}_{l} \in \mathscr{F}}\left(-\mathscr{T}_{\mathscr{S}_{i}}^{-2 l_{l}+\delta_{l}(\sigma)}\right)\right] Z(\alpha, p, z)\right]_{z_{a}=0}
\end{aligned}
$$

which is equal to (V.12) by Theorems $1-4$.

\section{Conclusion}

In this paper, using the parametric integral representation, we have defined a renormalized Feynman amplitude. The renormalized integrand is obtained by applying the product of substraction operators over all possible, whether connected, divergent, proper or not, families of parameters.

This product is "a priori" non commutative, and the integrand seems "a priori" oversubstracted. However this product is shown to be independent of the order of its factors, by simple generalization of Appelquist's theorem, so that the integrand is indeed integrable.

On the other hand, the complete product reduces to the product over divergent, connected, proper subgraphs only, and then is proved to be equal to a sum over Zimmermann's forests of renormalization parts.

Since this formulation is equivalent to Zimmermann's $R$-operation (up to a finite renormalization for theories with spins), it provides a new proof of the B.P.H. theorem (we did not consider the $\varepsilon \rightarrow 0$ limit in the propagator $\left.\frac{1}{k^{2}-m^{2}+i \varepsilon}\right)$.

Finally, as noted in the end of Section III, our substraction operator does not depend on the actual topology of the graph (but only on the total number of internal lines). The same property holds of course for any graph in a theory with spin particles and derivative couplings. Moreover, one can make further remarks:

Suppose that we introduce additional parameters $\alpha_{l+1}, \ldots, \alpha_{k}$ and the corresponding dilatation coefficients $\varrho_{i}\left(2^{l} \leqq i \leqq 2^{k}-1\right)$. Then, due to (A.I-13), (III.19) is equivalent to

$$
R_{l} Z=\prod_{1}^{2^{k}-1}\left(1-T_{\varrho_{l}}^{M-2 l_{l}}\right) \prod_{1}^{2^{k}-1} \varrho_{i}^{M} \tilde{Z}=R Z
$$


where now $R$ is independent of $l$ provided $l \leqq k$. Furthermore, we expect the $R$ substraction operator and the $\alpha$ integration to commute provided a cut-off has been introduced. Then the $R$ operator could be used to renormalize a Green's function truncated at a given number of internal lines.

We propose ourselves to examine this program in forthcoming publications, as well as cut-off expansions and scaling behaviour of Feynman graphs.

\section{Appendix I}

The $\mathscr{T}$-Operators

Given a $C^{\infty}$-function $f(x)$, the Taylor operator at $x=0, T^{n}$ is defined as

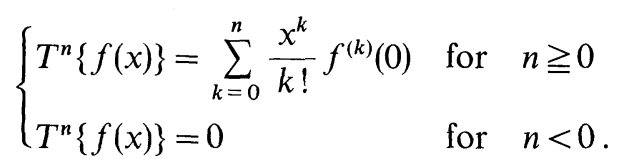

An important property of $T^{n}$ is: for any integer $\lambda \geqq 0$

$$
T^{n}\left\{x^{\lambda} f(x)\right\}=x^{\lambda} T^{n-\lambda}\{f(x)\} .
$$

We want to generalize the definition (A.I-1) to functions such that $x^{P} f(x)=g(x)$ is a $C^{\infty}$ function in $[0, a>0[$ for at least one value of the integer $p \gtrless 0$.

Definition. For any integer $n \gtrless 0$ and $x \in[0, a[$

$$
\mathscr{T}^{n}\{f(x)\}=\frac{1}{x^{\lambda}} T^{n+\lambda}\left\{x^{\lambda} f(x)\right\}
$$

where $\lambda$ is any integer greater than or equal to $p$.

We note the following properties:

1) Of course, definition (A.I-3) is independent of $\lambda \geqq p$. In particular

$$
\mathscr{T}^{n}\{f(x)\}=\frac{1}{x^{P}} T^{n+p}\left\{x^{P} f(x)\right\}
$$

2) $\mathscr{T}^{n}\{f(x)\}$ is a finite sum of terms the behaviour of which at $x=0$ goes from $\frac{1}{x^{P}}$ to $x^{n}$.

3) $\mathscr{T}^{n}\{f(x)\}=0$ for $n+p<0$.

4) $\left(1-\mathscr{T}^{n}\right)\{f(x)\}$ behaves at $x=0$ at least as $x^{q}$ with $q>n$.

5) $\left(1-\mathscr{T}^{n_{2}}\right) \mathscr{T}^{n_{1}}\{f(x)\}=0$ if $n_{2} \geqq n_{1}$. 
The integral representation for the rest of the Taylor expansion gives

$$
\left(1-\mathscr{T}^{n}\right)\{f(x)\}=\int_{0}^{1} d \xi \frac{(1-\xi)^{n+\lambda}}{(n+\lambda) !} \frac{\partial^{n+\lambda+1}}{\partial \xi^{n+\lambda+1}}\left(\xi^{\lambda} f(x \xi)\right)
$$

with $\lambda \geqq \operatorname{Sup}(p,-n)$.

Let us now look at the properties of a product of $\mathscr{T}$ 's acting on functions of several variables.

Given a $C^{\infty}$ function of several variables $f\left(x_{1}, \ldots, x_{k}\right)$ which behaves as $\frac{1}{x_{i}^{P_{i}}}$ at $x_{i}=0, x_{j \neq i} \neq 0$, with integers $p_{i} \gtrless 0$, we assume the conditions of (A.I-3) to be satisfied after an arbitrary number of $\mathscr{T}$ operations. Then, the following equation holds

$$
\mathscr{T}_{x_{1}}^{n_{1}} f=\frac{1}{x_{1}^{\lambda_{1}}} T_{x_{1}}^{n_{1}+\lambda_{1}}\left\{x_{1}^{\lambda_{1}} f\right\}=\frac{1}{x_{1}^{P_{1}}} \sum_{q=0}^{n_{1}+p_{1}} \frac{x_{1}^{q}}{q !} g^{(q)}\left(0, x_{2}, \ldots x_{k}\right)
$$

where $g^{(q)}$ is the $q^{\text {th }}$ derivative in regards to $x_{1}$ of $g=x_{1}^{P_{1}} f$ and $\lambda_{1} \geqq p_{1}$. Each of the $g^{(q)}$ derivatives in (A.I-9) behaves at $x_{2}=0, x_{j>2} \neq 0$ as $\frac{1}{x_{2}^{P_{2}+d_{2}(q)}}$ with integers $d_{2}(q) \gtrless 0$. We then define $\mathscr{T}_{x_{2}}^{n_{2}} \mathscr{T}_{x_{1}}^{n_{1}} f$ from (A.I-3) with $\lambda_{2} \geqq p_{2}+D_{2}$ where $D_{2}=\operatorname{Sup}_{q}\left\{d_{2}(q)\right\}$.

Finally,

$$
\mathscr{T}_{x_{k}}^{n_{k}} \ldots \mathscr{T}_{x_{1}}^{n_{1}} f=\prod_{i=1}^{k} x_{i}^{-\lambda_{i}} T_{x_{k}}^{n_{k}+\lambda_{k}} \ldots T_{x_{1}}^{n_{1}+\lambda_{1}}\left\{\prod_{i} x_{i}^{\lambda_{i}} f\right\}
$$

where $\lambda_{i} \geqq p_{i}+D_{i}$ and $D_{1}=0$.

It is clear from this definition that the $\mathscr{T}$ 's do not commute.

We observe the following properties:

1) Definition (A.I-10) is independent of the $\lambda_{i}$ 's provided that $\lambda_{i} \geqq p_{i}+D_{i}$

$$
\mathscr{T}_{x_{k}}^{n_{k}} \ldots \mathscr{T}_{x_{1}}^{n_{1}} f=\prod_{i=1}^{k} x_{i}^{-p_{t}-D_{\imath}} T_{x_{k}}^{n_{k}+p_{k}+D_{k}} \ldots T_{x_{1}}^{n_{1}+p_{1}}\left\{\prod_{i} x_{i}^{p_{i}+D_{\imath}} f\right\} .
$$

2) $\mathscr{T}_{x_{k}}^{n_{k}} \ldots \mathscr{T}_{x_{1}}^{n_{1}} f$ is a finite sum of terms the behaviour of which at $x_{i}=0, x_{j} \neq 0$ goes from $\frac{1}{x_{i}^{P_{i}+D_{\imath}}}$ to $x_{i}^{n_{i}}$.

3) $\mathscr{T}_{x_{k}}^{n_{k}} \ldots \mathscr{T}_{x_{1}}^{n_{1}} f=0$ if for at least one $x_{i}$ the condition $n_{i}+p_{i}+D_{i}<0$ is satisfied.

4) $\left(1-\mathscr{T}_{x_{k}}^{n_{k}}\right) \ldots\left(1-\mathscr{T}_{x_{1}}^{n_{1}}\right) f$ is expressed by a formula similar to (A.I-10) where $T$ is replaced by $1-T$ and $D_{i}$ by $D_{i}^{\prime}=\sup \left(0, D_{i}\right)$.

5) $\left(1-\mathscr{T}_{x_{k}}^{n_{k}}\right) \ldots\left(1-\mathscr{T}_{x_{1}}^{n_{1}}\right) f$ behaves at $x_{k}=0, x_{j \neq k}^{\neq} 0$ at least as $x_{k}^{q}$ 
with $q>n_{k}$ but nothing can be said about the behaviour in the other variables except in the case where the $(1-\mathscr{T})$ 's commute. In this case the above property is true for all $x_{i}$.

Example. We take

$$
\begin{array}{r}
f(x, y)=\frac{1}{x+y}, \\
\left(1-\mathscr{T}_{y}^{-1}\right)\left(1-\mathscr{T}_{x}^{0}\right) f=\frac{1}{x+y}
\end{array}
$$

which clearly does not behave as $x^{q}, q>0$ for $x=0, y \neq 0$.

$$
\text { 6) } \ldots\left(1-\mathscr{T}_{x_{i}}^{n_{i}}\right) \ldots \mathscr{T}_{x_{i}}^{n_{i}^{\prime}} \ldots f=0 \text { if } \quad n_{i}^{\prime} \leqq n_{i}
$$

whatever the $\mathscr{T}$ 's in between.

\section{Corollaries}

a) $\ldots\left(1-\mathscr{T}_{x_{i}}^{n_{i}}\right) \ldots f=\ldots\left(1-\mathscr{T}_{x_{i}}^{n_{i}}\right) \ldots\left(1-\mathscr{T}_{x_{i}}^{n_{i}^{\prime}}\right) \ldots f \quad$ if $\quad n_{i}^{\prime} \leqq n_{i}$

b) $\ldots \quad \mathscr{T}_{x_{i}}^{n_{i}^{\prime}} \ldots f=\ldots \quad \mathscr{T}_{x_{i}}^{n_{i}} \ldots \quad \mathscr{T}_{x_{i}}^{n_{i}^{\prime}} \ldots f$ if $n_{i}^{\prime} \leqq n_{i}$.

The corresponding representation for (A.I-8) gives

$$
\begin{aligned}
\left(1-\mathscr{T}_{x_{k}}^{n_{k}}\right) \ldots\left(1-\mathscr{T}_{x_{1}}^{n_{1}}\right) f=\int_{0}^{1} d \xi_{k} \ldots \int_{0}^{1} d \xi_{1} \\
\cdot \prod_{1}^{k}\left\{\frac{\left(1-\xi_{i}\right)^{n_{i}+\lambda_{i}}}{\left(n_{i}+\lambda_{i}\right) !} \frac{\partial^{n_{i}+\lambda_{i}+1}}{\partial \xi_{i}^{n_{i}+\lambda_{i}+1}}\right\}\left[\prod_{1}^{k} \xi_{i}^{\lambda_{i}} f\left(x_{i} \xi_{i}\right)\right] .
\end{aligned}
$$

Provided $\lambda_{i} \geqq \operatorname{Sup}\left(p_{i}+D_{i}^{\prime},-n_{i}\right)$, the above expression is always integrable by parts for a given order of integration. This order defines the order of application of the $(1-\mathscr{T})$ 's over $f$.

If we choose the $\lambda_{i}$ 's large enough so that the integrations by parts can be performed in any order, a sufficient but not necessary condition for the complete product of $(1-\mathscr{T})$ 's to commute is that the integral is absolutely convergent. This does not mean that the $\mathscr{T}$ 's commute. Such a situation will appear for Feynman integrands.

We now define the $\mathscr{T}$ operators relative to a family of variables. Given a function of several variables $f\left(x_{1} \ldots x_{k}\right)$ and a family of $x$ 's $\left\{x_{i_{1}} \ldots x_{i_{p}}\right\}$, we define

$$
\mathscr{T}_{\left\{x_{i_{1}}, \ldots, x_{i_{p}}\right\}}^{n} f=\left[\mathscr{T}_{\varrho}^{n} f\left(\varrho x_{i_{1}}, \ldots, \varrho x_{i_{p}}, x_{i_{p+1}}, \ldots, x_{i_{k}}\right)\right]_{\varrho=1} .
$$




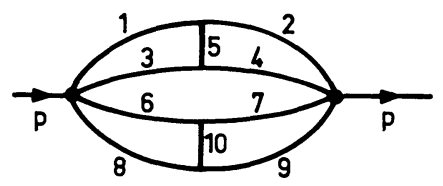

Fig. 1

The direct application of (A.I-8) gives

$$
\begin{aligned}
& \left(1-\mathscr{T}_{\left\{x_{1} \ldots x_{i_{p}}\right\}}^{n}\right) f \\
& =\int_{0}^{1} d \xi \frac{(1-\xi)^{n+\lambda}}{(n+\lambda) !} \frac{\partial^{n+\lambda+1}}{\partial \xi^{n+\lambda+1}}\left[\xi^{\lambda} f\left(x_{i_{1}} \xi, \ldots x_{i_{p}} \xi, x_{i_{p}+1}, \ldots x_{i_{k}}\right)\right]
\end{aligned}
$$

with $\lambda \geqq \operatorname{Sup}(p,-n)$ and $p$ denotes the order of the pole in the $\xi$ variable of the function $f$.

In this paper we deal with functions $f=\frac{e^{\varphi\left(\alpha_{t}\right)}}{P^{k}\left(\alpha_{i}\right)}$ where the function $\varphi\left(\alpha_{i}\right)$ displays no singularities relative to any family of $\alpha$ 's; the function $f$ develops poles due to zeros of the (homogeneous) polynomial $P$ for any family of $\alpha$ 's associated with a diagram with loops.

The product of $\mathscr{T}$ 's operating upon $f$ always commutes as long as the families of $\alpha$ 's do not overlap (see the end of Section II for the definition of overlapping families). If two families of $\alpha$ 's $\mathscr{S}_{1}$ and $\mathscr{S}_{2}$ overlap and if $L_{\mathscr{S}_{1} \cup \mathscr{S}_{2}}=L_{\mathscr{S}_{1}}+L_{\mathscr{S}_{2}}-L_{\mathscr{S}_{1} \cap \mathscr{S}_{2}}$ where the $L$ 's refer to the corresponding number of loops, $\mathscr{T}_{\mathscr{S}_{1}}^{n_{1}} \mathscr{T}_{\mathscr{H}_{2}}^{n_{2}} f=\mathscr{T}_{\mathscr{S}_{2}}^{n_{2}} \mathscr{T}_{\mathscr{S}_{1}}^{n_{1}} f$; but if $L_{\mathscr{S}_{1} \cup \mathscr{S}_{2}}>L_{\mathscr{S}_{1}}+L_{\mathscr{S}_{2}}$ $-L_{\mathscr{C}_{1} \cap \mathscr{S}_{2}}$, the $\mathscr{T}$ 's generally do not commute: this is the main technical difficulty of this paper; it was avoided by Appelquist [4] who considered only renormalizable theories in four dimensions, and Green's functions with two or more external legs.

Example. Consider the graph in Fig. 1 with a space-time dimension $D=5$, and take $\mathscr{S}_{1}=\left\{\alpha_{1}, \alpha_{2}, \alpha_{3}, \alpha_{4}, \alpha_{5}\right\}, \mathscr{S}_{2}=\left\{\alpha_{6}, \alpha_{7}, \alpha_{8}, \alpha_{9}, \alpha_{10}\right\}$. For this graph:

$$
\begin{aligned}
& P=\left(\alpha_{1}+\alpha_{2}+\alpha_{3}+\alpha_{4}\right) \cdot \alpha_{5} \cdot\left[\alpha_{10}\left(\alpha_{6}+\alpha_{7}\right)\left(\alpha_{8}+\alpha_{9}\right)+\alpha_{6} \alpha_{8}\left(\alpha_{7}+\alpha_{9}\right)\right. \\
& \left.+\alpha_{7} \alpha_{9}\left(\alpha_{8}+\alpha_{6}\right)\right]+\left(\alpha_{1}+\alpha_{3}\right)\left(\alpha_{2}+\alpha_{4}\right)\left[\left(\alpha_{6}+\alpha_{7}\right)\left(\alpha_{8}+\alpha_{9}\right) \cdot \alpha_{10}\right. \\
& \left.+\alpha_{6} \alpha_{7}\left(\alpha_{8}+\alpha_{9}\right)+\alpha_{8} \alpha_{9}\left(\alpha_{6}+\alpha_{7}\right)\right]+\left(\alpha_{1}, \alpha_{2}, \alpha_{3}, \alpha_{4}, \alpha_{5} \hookleftarrow \alpha_{6}, \alpha_{7}, \alpha_{8}, \alpha_{9}, \alpha_{10}\right) .
\end{aligned}
$$

Then

$$
\mathscr{T}_{\mathscr{S}_{2}}^{-10} \cdot \mathscr{T}_{\mathscr{S}_{1}}^{-10} \frac{e^{-i p_{i} d_{\overline{i j}}^{1} p_{J}}}{P^{5 / 2}}=\frac{1}{\tilde{P}^{5 / 2}}
$$


where $\tilde{P}$ is the sum of the two first terms in the right hand side of (A.I-18). $\mathscr{T}_{\mathscr{S}_{1}}^{-10} \mathscr{T}_{\mathscr{S}_{2}}^{-10} Z$ is obtained from $\mathscr{T}_{\mathscr{S}_{2}}^{-10} \mathscr{T}_{\mathscr{S}_{1}}^{-10} . Z$ by exchanging $\alpha_{1}$ to $\alpha_{5}$ into $\alpha_{6}$ to $\alpha_{10}$ respectively. This example shows that $L_{\mathscr{S}_{1} \cup \mathscr{S}_{2}}>L_{\mathscr{S}_{1}}+L_{\mathscr{S}_{2}}$ $-L_{\mathscr{S}_{1} \cap \mathscr{S}_{2}}$ implies the non commutativity of $\mathscr{T}_{\mathscr{S}_{1}}$ and $\mathscr{T}_{\mathscr{S}_{2}}$.

\section{Appendix II \\ Commutativity of the Complete Product $\prod_{i}\left(1-\mathscr{T}_{\mathscr{P}_{\imath}}^{-2 l_{\imath}+\delta_{\imath}}\right) Z$}

Section II is mainly based on the fact that the complete product of $(1-\mathscr{T})$ 's does not depend on their order. This Appendix is devoted to the proof of this important property in the general case with spin and derivatives couplings.

Since $Z(\alpha, p, z)$ defined in (V.4) is a regular function of $z$, we can write

$$
\begin{aligned}
& \prod_{i}\left(1-\mathscr{T}_{\mathscr{S}_{\imath}}^{-2 l_{i}}\right) \prod_{a=1}^{v}\left(\frac{1}{i \sqrt{\alpha_{a}}} \frac{\partial}{\partial z_{a}}\right) Z(\alpha, p, z) \\
= & \prod_{a=1}^{v}\left(\frac{1}{i \sqrt{\alpha_{a}}} \frac{\partial}{\partial z_{a}}\right) \Pi\left(1-\mathscr{T}_{\mathscr{S}_{l}}^{-2 l_{l}+\delta_{l}(\sigma)}\right) Z
\end{aligned}
$$

where $\delta_{i}$ is the total number of derivatives $\frac{\partial}{\partial z}$ attached to the lines of $\mathscr{S}_{i}$. By formula (A.I-15), the commutativity of the product is established if the integral

$\int_{0}^{1} \cdots \int_{0}^{1} d \xi_{1} \ldots d \xi_{k} \prod_{1}^{k} \frac{\left(1-\xi_{i}\right)^{-2 l_{l}+\delta_{i}+\lambda_{i}}}{\left(-2 l_{i}+\delta_{i}+\lambda_{i}\right) !} \frac{\partial^{-2 l_{i}+\delta_{i}+\lambda_{l}+1}}{\partial \xi_{i}^{-2 l_{i}+\delta_{l}+\lambda_{i}+1}}\left\{\prod_{1}^{k} \xi_{i}^{\lambda_{i}} \tilde{Z}\right\}$

is absolutely convergent. $\tilde{Z}$ is the expression $Z$ where each $\sqrt{\alpha_{j}}$ is dilated by all $\xi_{i}$ 's such that $\alpha_{j} \in \mathscr{S}_{i}$.

The absolute convergence of the above integral was already proved by Appelquist [4]. Actually, his demonstration was performed on the complete integral $\int_{0}^{\infty} d \alpha \ldots \int_{0}^{1} d \xi \ldots$ where the $\xi$ 's refer to proper divergent subgraphs. The generalization to all families of $\alpha$ 's is trivial and we shall content ourselves with mentioning the most important steps.

We first perform the derivatives $\frac{\partial}{\partial \xi_{i}}$ in (A.II-2) and we obtain a sum of terms of the form

$$
\prod_{i=1}^{k_{1}} \xi_{i}^{-D L_{l}+2 l_{l}-\delta_{t}+p_{t}-1} \frac{\partial^{p_{t}}}{\partial \xi_{i}^{p_{i}}} \prod_{j=1}^{k_{2}} \xi_{j}^{p_{j}} \frac{\partial^{D L_{J}-2 l_{J}+\delta_{j}+p_{j}+1}}{\partial \xi_{j}^{D L_{j}-2 l_{J}+\delta_{j}+p_{j}+1}} \tilde{Z}^{\prime}
$$


where

$$
\tilde{Z}^{\prime}=\prod_{i}^{k} \xi_{i}^{L_{i} D} \tilde{Z} .
$$

In (A.II-3), the indices $i$ (resp. $j$ ) from 1 to $k_{1}$ (resp. $k_{2}$ ) refer to families of $\alpha$ 's such that $D L_{i}-2 l_{i}+\delta_{i} \leqq-1\left(\right.$ resp. $\left.D L_{j}-2 l_{j}+\delta_{j}>-1\right) ; p_{i}\left(\right.$ resp. $\left.p_{j}\right)$ are integers which take any value from 0 to $-2 l_{i}+\delta_{i}+\lambda_{i}+1$ (resp. $\left.\lambda_{j}-D L_{j}\right)$. The absolute convergence is proved if for any family $\left\{\xi_{1}, \ldots, \xi_{r}\right\}$ the expression (A.II-3) has a pole of order less than $r$ in $\xi_{1}=\cdots=\xi_{r}=0$.

Given a family $\left\{\xi_{1}, \ldots \xi_{r}\right\}$ corresponding to the families of $\alpha$ 's $\mathscr{S}_{1}, \ldots \mathscr{S}_{r}$, we define the nested families of $\alpha$ 's

$$
\mathscr{S}(r) \subset \mathscr{S}(r-1) \subset \cdots \subset \mathscr{S}(1)
$$

where $\mathscr{S}(i)$ is the set of $\alpha$ 's which are contained in at least $i$ of the families $\mathscr{S}_{1}, \ldots, \mathscr{S}_{r}$. The number of lines, loops, and derivatives $\frac{\partial}{\partial z}$ of $\mathscr{S}(i)$ (resp. $\mathscr{S}_{i}$ ) is denoted by $l(i), L(i)$ and $\delta(i)\left(\right.$ resp. $l_{i}, L_{i}$ and $\left.\delta_{i}\right)$. We have the following properties:

$$
\begin{aligned}
& \sum_{1}^{r} l_{i}=\sum_{1}^{r} l(i) \\
& \sum_{1}^{r} \delta_{i}=\sum_{1}^{r} \delta(i) .
\end{aligned}
$$

We introduce the following polynomials in $\xi$ and $\sqrt{\alpha}: \tilde{P}^{\prime}, \tilde{N}_{i j}^{\prime}, \tilde{N}_{i a}^{\prime}$ and $\tilde{N}_{a b}^{\prime}$. They are deduced from the polynomials $P$ and $N_{i j}$ defined in Section I, and from the polynomials $N_{i a}$ and $N_{a b}$ defined in Section V as $\tilde{Z}^{\prime}$ was deduced from $Z$; for instance

$$
\tilde{P}^{\prime}=\prod_{1}^{k} \xi_{i}^{-2 L_{i}} \tilde{P}(\sqrt{\alpha}, \xi) .
$$

Then, each term of these polynomials has a zero of order $m$ at $\xi_{1}=\cdots=\xi_{r}=0$ with

$$
m \geqq m_{0}=\sum_{1}^{r}\left(2 L(i)-2 L_{i}\right) .
$$

Moreover there exists in polynomial $\tilde{P}^{\prime}$ at least one term such that $m=m_{0}$. Now, it is easy to convince oneself that

$$
\frac{\partial^{n_{1}}}{\partial \xi_{1}} \ldots \frac{\partial^{n_{k}}}{\partial \xi_{k}}\left\{\tilde{P}^{\prime}, \tilde{N}_{i_{J}}^{\prime}, \tilde{N}_{i a}^{\prime}, \tilde{N}_{a b}^{\prime}\right\}
$$


is a sum of terms, each of them develop a zero of order $2 L_{j}+x_{j}$ when all $\sqrt{\alpha}$ 's of any family vanish; $x_{j}$ is greater or equal to $n_{j}$ and depends upon each term; $x_{j}-n_{j}$ is the order of the zero of the corresponding term when $\xi_{j}$ vanishes.

In (A.II-3),

$$
\prod_{i=1}^{k_{1}} \frac{\partial^{p_{i}}}{\partial \xi_{i}} \prod_{j=1}^{k_{2}} \frac{\partial^{D L_{j}-2 l_{j}+\delta_{j}+p_{j}+1}}{\partial \xi_{j}} \tilde{Z}^{\prime}=\sum_{\{\sigma\}} \frac{R_{\sigma}}{\tilde{P}^{\prime D / 2+\sigma}}
$$

where $R_{\sigma}$ is a sum of products of $\sigma$ terms of the form (A.II-10), times the exponential. Each term in $R_{\sigma}$ has thus a zero of order $2 \sigma L_{i}+x_{i}$ when all $\sqrt{\alpha}$ 's belonging to $\mathscr{S}_{i}$ vanish, with

$$
x_{i} \geqq p_{i} \quad \text { if } \quad D L_{i}-2 l_{i}+\delta_{i} \leqq-1,
$$

and $x_{i} \geqq D L_{i}-2 l_{i}+\delta_{i}+p_{i}+1$ otherwise. Similarly, each term in $R_{\sigma}$ has a zero of order $2 \sigma L(i)+x(i)$ when all $\sqrt{\alpha}$ 's belonging to $\mathscr{S}(i)$ vanish, with $x(i) \geqq D L(i)-2 l(i)+\delta(i)+1$ (for non empty $\mathscr{S}(\mathrm{i})$ ).

The structure of the families $\mathscr{S}_{i}$ and $\mathscr{S}(i)$ is such that

$$
\sum_{i=1}^{r}\left(2 \sigma L_{i}+x_{i}\right)=\sum_{i=1}^{r}[2 \sigma L(i)+x(i)] .
$$

Finally, each term in $R_{\sigma}$ has a zero of order:

$$
\sum_{1}^{k_{1}}\left(x_{i}-p_{i}\right)+\sum_{1}^{k_{2}}\left(x_{i}+2 l_{i}-D L_{i}-\delta_{i}-p_{i}-1\right)
$$

when $\xi_{1}=\cdots=\xi_{r}=0$. Combining (A.II-13), (A.II-12), (A.II-6) and (A.II-7), and the value of $m_{0}$, it is found that expression (A.II-3) has a pole of order less than $r$ when $\xi_{1}=\cdots=\xi_{r}=0$ and the absolute convergence is ensured.

\section{Appendix III}

\section{Demonstration of Equation (III-6)}

We use here, the same notations as in Section III, Theorem 1; we intend to prove

$$
\prod_{i \geqq n+1}\left(1-\mathscr{T}_{\mathscr{P}_{l}}^{-2 l_{l}}\right)\left(-\mathscr{T}_{\mathscr{S}_{n}}^{-2 l}\right)\left[\sum_{\mathscr{F} \in \mathscr{S}_{n-1}^{\prime}} \prod_{\mathscr{S}_{j} \in \mathscr{F}}\left(-\mathscr{T}_{\mathscr{S}_{j}}^{-2 l_{j}}\right) Z\right]=0
$$

where $\mathscr{E}_{n-1}^{\prime}$ is the set of forests containing at least one element overlapping with $\mathscr{S}_{n}$.

Let us consider a forest $\mathscr{F} \cup \mathscr{F}^{\prime}$ of $\mathscr{E}_{n-1}^{\prime}: \mathscr{F}^{\prime}$ (resp. $\mathscr{F}$ ) is the subforest of elements overlapping (resp. non overlapping) with $\mathscr{S}_{n}, \mathscr{F}^{\prime}$ is never empty. 


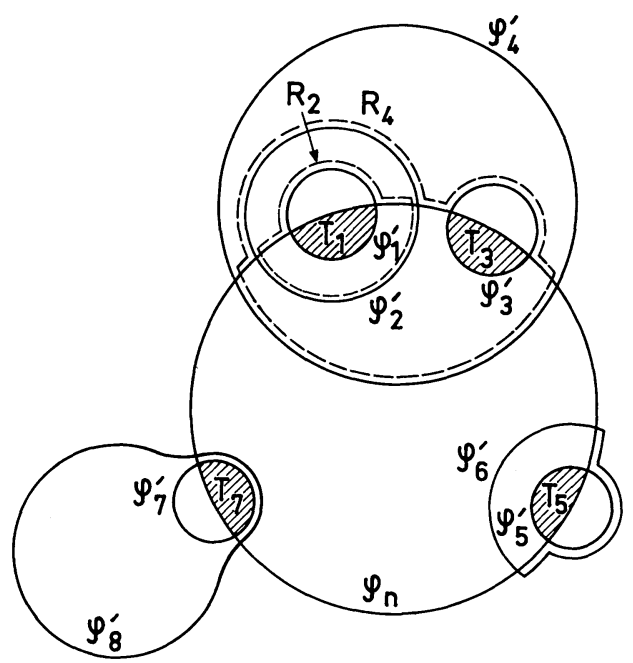

Fig. 2. Construction of the families $R_{l}$ and $T_{l}$, from the families $\mathscr{S}_{i}^{\prime}$ overlapping with $\mathscr{S}_{n}$. The stripped areas are the $T_{\imath}$ 's. The dashed lines surround

and

$$
R_{2}=\mathscr{S}_{2}^{\prime} \cap\left(\mathscr{S}_{n} \cup \mathscr{S}_{1}^{\prime}\right)
$$

$$
R_{4}=\mathscr{S}_{4}^{\prime} \cap\left(\mathscr{S}_{n} \cup \mathscr{S}_{2}^{\prime} \cup \mathscr{S}_{3}^{\prime}\right)
$$

Note that $R_{6}=\mathscr{S}_{6}^{\prime}$ and $R_{8}=\mathscr{S}_{7}^{\prime}$.

$\mathscr{S}_{i}^{\prime}$ denote the elements of $\mathscr{F}^{\prime}$. In $\mathscr{F}^{\prime}$ we call minimal (resp. maximal) element, an element which does not contain (resp. which is not contained in) any other element of $\mathscr{F}^{\prime}$.

To any minimal element $\mathscr{S}_{i}^{\prime}$ we associate the family

$$
T_{i}=\mathscr{S}_{n} \cap \mathscr{S}_{i}^{\prime} .
$$

To any non minimal element $\mathscr{S}_{i}^{\prime}$, let $I_{i}$ be the set of indices $k$ such that $\mathscr{S}_{k}^{\prime} \subset \mathscr{S}_{i}^{\prime}$ and $\mathscr{S}_{k}^{\prime}$ is maximal in $\mathscr{S}_{i}^{\prime}$. Then we define:

$$
R_{i}=\mathscr{S}_{i}^{\prime} \cap\left(\mathscr{S}_{n} \bigcup_{k \in I_{i}} \mathscr{S}_{k}^{\prime}\right)=\left(\mathscr{S}_{i}^{\prime} \cap \mathscr{S}_{n}\right) \bigcup_{k \in I_{2}} \mathscr{S}_{k}^{\prime}
$$

(some intersections might be a set of vertices with no lines; some $R_{i}$ might be $\mathscr{S}_{i}^{\prime}$ or even $\left.\bigcup_{k \in I_{i}} \mathscr{S}_{k}^{\prime}\right)$. We note that the $T_{i}^{\prime}$ s are contained in $\mathscr{S}_{n}$, whereas the $R_{i}$ 's overlap with $\mathscr{S}_{n}$.

On Fig. 2, the construction of the families $R_{i}$ and $T_{i}$ is illustrated: each bubble in Fig. 2 represents a set of lines and vertices. 
Lemma 1. Let us consider $U=\mathscr{S}_{n} \cup \mathscr{S}_{1}^{\prime} \cup \cdots \cup \mathscr{S}_{k}^{\prime}$ :

The elements of $\mathscr{F} \cup \mathscr{F}^{\prime}$, the $R_{i}$ 's, the $T_{i}$ 's form a forest in $\mathscr{E}_{n-1}^{\prime} ; U$ form a forest with them.

We let the reader to convince himself of this property.

Lemma 2. The different numbers of lines are related by:

$$
l_{U}=l_{\mathscr{S}_{n}}+\sum_{\mathscr{S}_{i}^{\prime} \in \mathscr{F}^{\prime}} l_{\mathscr{S}_{i}^{\prime}}-\sum_{i} l_{R_{i}}-\sum_{i} l_{T_{i}}
$$

where $\sum_{i}$ means a sum over all $R_{i}$ 's and all $T_{i}$ 's.

The proof is based upon the trivial identity

$$
l_{G_{1} \cup G_{2}}=l_{G_{1}}+l_{G_{2}}-l_{G_{1} \cap G_{2}} .
$$

If we apply (A.III-5) to $U$,

$$
l_{U}=l_{\mathscr{S}_{n}}+\sum_{\mathscr{S}_{i}^{i} \max }\left(l_{\mathscr{S}_{i}^{i}}-l_{\mathscr{S}_{n} \cap \mathscr{S}_{i}}\right) .
$$

Now, if $\mathscr{S}_{i}^{\prime}$ is a minimal element,

$$
-l_{\mathscr{S}_{n} \cap \mathscr{S}_{i}}=-l_{T_{i}}
$$

otherwise from (A.III-3)

$$
-l_{\mathscr{S}_{n} \cap \mathscr{S}_{i}^{i}}=-l_{R_{\imath}}+\sum_{k \in I_{\imath}}\left(l_{\mathscr{S}_{k}}-l_{\mathscr{S}_{n} \cap \mathscr{S}_{k}^{\prime}}\right) .
$$

The iterated application of (A.III-8) up to a minimal element, in (A.III-6) leads to

$$
l_{U}=l_{\mathscr{S}_{n}}+\sum_{\mathscr{S}_{i}^{\prime} \text { non min }}\left(l_{\mathscr{S}_{i}^{\prime}}-l_{R_{i}}\right)+\sum_{\mathscr{S}_{i}^{\prime} \min }\left(l_{\mathscr{S}_{i}^{\prime}}-l_{T_{i}}\right)
$$

which is equivalent to (A.III-4).

To go further, we need to introduce some new concepts. Let us denote by $\Omega$ the operation constructing $R_{i}$ from $\mathscr{S}_{i}^{\prime}$ and the set of $\mathscr{S}_{k}^{\prime}$ 's in (A.III-3)

$$
\Omega\left(\mathscr{S}_{i}^{\prime} ;\left\{\mathscr{S}_{k}^{\prime}\right\}\right)=R_{i}=\mathscr{S}_{i}^{\prime} \cap\left(\mathscr{S}_{n} \bigcup_{k \in I_{i}} \mathscr{S}_{k}^{\prime}\right)
$$

we also use the notation $\Omega\left(\mathscr{F}^{\prime}\right)$ for the set of all $R_{i}$ 's constructed in (A.III-10). We consider the forest $\mathscr{G}$ of elements overlapping with $\mathscr{S}_{n}$ :

$$
\mathscr{G}=\mathscr{F}^{\prime} \cup \Omega\left(\mathscr{F}^{\prime}\right) .
$$

There exist several forests $\mathscr{F}^{\prime}$ such that (A.III-11) generates the same forest $\mathscr{G} ; \mathscr{G}$ itself belongs to this set since

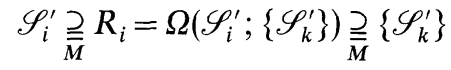


where the symbol $\underset{\bar{M}}{\supseteqq}$ means that each element at the right of $\underset{\bar{M}}{\supseteqq}$ is maximal in the element at the left, implies

$$
\Omega\left(\mathscr{S}_{i}^{\prime} ; R_{i}\right)=\Omega\left(R_{i} ;\left\{\mathscr{S}_{k}^{\prime}\right\}\right)=R_{i} .
$$

We intend to find out the unique element $\mathscr{F}^{\prime-}$ of the set, contained in all the other $\mathscr{F}^{\prime}$ 's of the set.

We define the subforest $\mathscr{H}$ of $\mathscr{G}$ as the forest of elements $\mathscr{X}_{i}$ such that: $\left.\mathscr{X}_{j} \supset \mathscr{X}_{i} \supset \mathscr{X}_{k}\right\}, \mathscr{X}_{i}$ is neither maximal nor minimal in $\mathscr{G}$ but is the only maximal element in $\mathscr{X}_{J}$, and

$$
\Omega\left(\mathscr{X}_{j} ; \mathscr{X}_{i}\right)=\Omega\left(\mathscr{X}_{i} ;\left\{\mathscr{X}_{k}\right\}\right)=\mathscr{X}_{i}
$$

Then $\mathscr{F}^{\prime-}$ is the complement of $\mathscr{H}$ in $\mathscr{G}$. Indeed, if $\mathscr{X}_{i}$ belongs to $\mathscr{H}$, then $\mathscr{X}_{j}$ and $\mathscr{X}_{k}$ 's in (A.III-14) belong to $\mathscr{F}^{\prime-} ; \mathscr{X}_{i}$ can be reconstructed from $\mathscr{X}_{j}$ and the $\mathscr{X}_{k}$ 's by the $\Omega$ operation:

$$
\mathscr{X}_{i}=\Omega\left(\mathscr{X}_{j} ;\left\{\mathscr{X}_{k}\right\}\right)
$$

and consequently $\mathscr{F}^{\prime-}$ generates $\mathscr{G}$ by (A.III-11). On the other hand, for any forest $\mathscr{F}^{\prime}$, the elements of $\Omega\left(\mathscr{F}^{\prime}\right)$ which are not in $\mathscr{F}^{\prime}$ are certainly in $\mathscr{H}$; then $\mathscr{F}^{\prime-}$ is contained in $\mathscr{F}^{\prime}$.

Example. Suppose that we consider in the graph of Fig. 3 the families:

$$
\begin{gathered}
\mathscr{S}_{n}=\left\{\alpha_{3}, \alpha_{4}, \alpha_{6}, \alpha_{7}, \alpha_{8}\right\}, \\
\mathscr{S}_{1}^{\prime}=\left\{\alpha_{1}, \alpha_{2}\right\}, \quad \mathscr{S}_{2}^{\prime}=\left\{\alpha_{1}, \alpha_{2}, \alpha_{6}\right\}, \\
\mathscr{S}_{3}^{\prime}=\left\{\alpha_{1}, \alpha_{2}, \alpha_{5}, \alpha_{6}\right\} \quad \text { and } \mathscr{S}_{4}^{\prime}=\left\{\alpha_{1}, \alpha_{2}, \alpha_{5}, \alpha_{6}, \alpha_{7}\right\} .
\end{gathered}
$$

Then $U$ is the complete graph and

$$
\begin{aligned}
& \mathscr{F}^{\prime}=\mathscr{Y}=\left\{\mathscr{S}_{1}^{\prime}, \mathscr{S}_{2}^{\prime}, \mathscr{S}_{3}^{\prime}, \mathscr{S}_{4}^{\prime}\right\} \\
& \mathscr{F}^{\prime-}=\left\{\mathscr{S}_{1}^{\prime}, \mathscr{S}_{3}^{\prime}, \mathscr{S}_{4}^{\prime}\right\}, \mathscr{H}=\left\{\mathscr{S}_{2}^{\prime}\right\} \quad \text { and } \quad \Omega\left(\mathscr{F}^{\prime-}\right)=\left\{\mathscr{S}_{2}^{\prime}, \mathscr{S}_{4}^{\prime}\right\} .
\end{aligned}
$$

To any forest $\mathscr{F} \cup \mathscr{F}^{\prime}$ in $\mathscr{E}_{n-1}^{\prime}$, we can associate the disjoint forests $\mathscr{F}^{\prime-}, \mathscr{H}, \mathscr{T} \equiv\left\{T_{i}\right\}$, and $\mathscr{F}^{-} \equiv \mathscr{F}-(\mathscr{T} \cap \mathscr{F})$ and we group several terms of (A.III-1) to form the expression ${ }^{4}$

$$
\begin{gathered}
\prod_{i \geqq n+1}\left(1-\mathscr{T}_{\mathscr{S}_{i}}\right)\left(-\mathscr{T}_{\mathscr{S}_{n}}\right) \prod_{\mathscr{F}-}\left(-\mathscr{T}_{\mathscr{S}_{i}}\right) \prod_{\mathscr{F}^{\prime}-}\left(-\mathscr{T}_{\mathscr{S}_{i}}\right) \\
\cdot \prod_{\mathscr{H}}\left(1-\mathscr{T}_{R_{\imath}}\right) \prod_{\mathscr{T}}\left(1-\mathscr{T}_{T_{i}}\right) Z .
\end{gathered}
$$

Each of those expressions is characterized by its $\left(\mathscr{F}^{\prime-}, \mathscr{F}^{-}\right)$.

\footnotetext{
${ }^{4}$ Some $T_{i}$ 's might be a set of vertices with no lines; then by definition $1-\mathscr{T}_{T}=1$.
} 


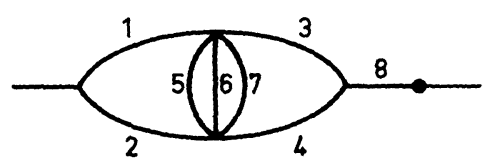

Fig. 3

Lemma 3. i) each term in (A.III-16) belongs to (A.III-1),

ii) each term in (A.III-1) is contained in one and only one expression (A.III-16).

This lemma is now a trivial consequence of the above definitions.

Lemma 4. Each expression (A.III-16) vanishes.

To prove this lemma, we only need to perform the $\mathscr{T}$ operations over a part of $\mathscr{F}^{\prime-}$ :

$$
\mathscr{K}=\mathscr{F}^{\prime-}-\left(\mathscr{F}^{\prime-} \cap \Omega\left(\mathscr{F}^{\prime-}\right)\right)
$$

that is the elements of $\mathscr{F}^{\prime-}$ which are not $R_{i}$ 's. It is easy to see that $\mathscr{K}$ is never empty. Moreover we have

$$
\begin{aligned}
\mathscr{H} & =\Omega(\mathscr{K}) \\
U & =\mathscr{S}_{n} \bigcup_{\mathscr{K}} \mathscr{S}_{i}^{\prime}
\end{aligned}
$$

and the $T_{i}$ 's constructed from $\mathscr{K}$ are the same as those constructed from $\mathscr{F}^{\prime-}$. In the above example (Fig. 3) $\mathscr{K}=\left\{\mathscr{S}_{1}^{\prime}, \mathscr{S}_{3}^{\prime}\right\}$.

From (A.III-4) applied to the forest $\mathscr{K}$, we obtain:

$$
l_{U}=l_{\mathscr{S}_{n}}+\sum_{\mathscr{K}} l_{\mathscr{S}_{i}}-\sum_{\mathscr{H}} l_{R_{i}}-\sum_{\mathscr{T}} l_{T_{i}}
$$

We now perform the different dilatations over the corresponding $\sqrt{\alpha}$ 's: we dilate the $\sqrt{\alpha}$ 's belonging to $U=\mathscr{S}_{n} \bigcup_{\mathscr{K}} \mathscr{S}_{i}^{\prime}$ by $\lambda$, to $\mathscr{S}_{n}$ by $\sigma$, to $T_{i} \in \mathscr{T}$ by $\tau_{i}$, to $R_{i} \in \mathscr{H}$ by $\varrho_{i}$ and to $\mathscr{P}_{i}^{\prime} \in \mathscr{K}$ by $\sigma_{i}$.

If we realize a partition of $\cup$ with the elements:

1) $T_{i}, R_{i}-\bigcup_{K \in I_{i}} \mathscr{S}_{K}^{\prime}, U-\bigcup_{i}\left(\mathscr{S}_{n} \cap \mathscr{S}_{i}^{\prime}\right)$ which are contained in $\mathscr{S}_{n}$.

2) $\mathscr{S}_{i}^{\prime}-T_{i}, \mathscr{S}_{j}^{\prime}-R$ which have no common lines with $\mathscr{S}_{n}$ it is easy to convince oneself that

$$
Z=Z\left(\lambda \sigma_{i} \prod_{\mathscr{S}_{j}^{\prime} \supset \mathscr{L}_{i}^{\prime}}\left(\varrho_{j} \sigma_{j}\right), \tau_{i} \sigma, \varrho_{i} \sigma, \lambda \sigma\right)
$$


The expression (A.III-16), is, by (A.I-13), equal to

$$
\begin{gathered}
\prod_{i \geqq n+1}\left(1-\mathscr{T}_{\mathscr{S}_{i}}\right)\left(-\mathscr{T}_{\mathscr{S}_{n}}\right) \prod_{\mathscr{F}-}\left(-\mathscr{T}_{\mathscr{S}_{i}}\right) \prod_{\mathscr{F}^{\prime}-}\left(-\mathscr{T}_{\mathscr{S}}\right)\left(1-\mathscr{T}_{U}\right) \\
\cdot \prod_{\mathscr{H}}\left(1-\mathscr{T}_{R_{i}}\right) \prod_{\mathscr{T}}\left(1-\mathscr{T}_{T_{i}}\right) \prod_{\mathscr{K}}\left(-\mathscr{T}_{\mathscr{S}_{i}}\right) Z
\end{gathered}
$$

where the $\mathscr{T}$ 's written at the right of $\mathscr{S}_{n}$ are all commutative. The above expression is the precise generalization to a complete forest of expression (III-8).

The product $\prod_{\mathscr{K}}\left(-\mathscr{T}_{\mathscr{S}_{i}}\right) Z$ is a finite sum of terms:

$$
\prod_{\mathscr{K}}\left(-\mathscr{T}_{\mathscr{S}_{i}^{\prime}}^{-2 l_{i}}\right) Z=\sum_{\left\{k_{k}\right\}} \prod_{i}\left(\lambda \sigma_{i} \prod_{\mathscr{S}_{j}^{\prime} \supset \mathscr{S}_{i}^{\prime}}\left(\varrho_{j} \sigma_{j}\right)\right)^{k_{i}} Z_{\left\{k_{i}\right\}}\left(\tau_{j} \sigma, \varrho_{j} \sigma, \lambda \sigma\right)
$$

with

$$
k_{i}+\sum_{\mathscr{S}_{j}^{\prime} \subset \mathscr{S}_{i}} k_{J} \leqq-2 l_{\mathscr{S}_{i}^{\prime}}
$$

Then,

$$
\left(1-\mathscr{T}_{U}\right) \prod_{\mathscr{H}}\left(1-\mathscr{T}_{R_{\imath}}\right) \prod_{\mathscr{T}}\left(1-\mathscr{T}_{T_{i}}\right) \prod_{\mathscr{K}}\left(-\mathscr{T}_{\mathscr{S}_{i}}\right) Z
$$

is a finite sum of terms; each term behaves in $\sigma$ at least as a power greater than

$$
-\sum_{i} 2 l_{T_{t}}-\sum_{i} 2 l_{R_{i}}-\sum_{i}\left[k_{i}+\sum_{\mathscr{S}_{J}^{\prime} \subset \mathscr{S}_{i}} k_{j}\right]-2 l_{U}
$$

which by (A.III-20) and (A.III-24) is greater or equal to $-2 l_{\mathscr{S}_{n}}$.

The application of the $\mathscr{T}^{\prime}$ 's in $\mathscr{F}^{-}$and $\left(\mathscr{F}^{\prime-}-\mathscr{K}\right)$ in (A.III-22) could only increase this power; finally, $\left(-\mathscr{T}_{\mathscr{S}_{n}}\right)$ causes all terms in (A.III-22) to vanish.

This completes the proof of (A.III-1).

Acknowledgments. We wish to express our gratitude to Professor R. Stora and to A. Voros for helpful discussions.

\section{References}

1. Bogoliubov, N. N., Parasiuk, O.: Acta Math. 97, 227 (1957)

Bogoliubov, N.N., Shirkov, D.W.: Introduction to the theory of quantized fields. New York: Interscience Publ. 1959

2. Hepp, K.: Commun. math. Phys. 2, 301-326 (1966)

3. Epstein, H., Glaser, V.: In: Statistical mechanics and quantum field theory, Les Houches 1970. New York: Gordon and Breach

4. Appelquist, Th.: Ann. Phys. 54, 27 -61 (1969) 
5. Zimmermann, W.: Commun. math. Phys. 15, 208-234 (1969)

Zimmermann, W.: In: Elementary particles and quantum field theory, Brandeis 1970. M.I.T. Press 1970

6. See for example:

Nakanishi, N.: Prog. Th. Phys. Suppl. 18, 1-81 (1961)

Todorov, I.T.: Analytic properties of Feynman diagrams in quantum field theory. Pergamon Press 1971

Further references may be found in these publications

Note Added in Proof. As pointed out recently by S. A. Anikin, M. K. Polivanov and O. I. Zavialov (Dubna preprint E2-7433), power counting arguments used in App. II and in the integrability of (III-1) are incomplete.

However, an algebraic proof of Eq. (III-3) is given by M. C. Bergère and Y. M. P. Lam (Berlin preprint TH I FU No. 3) for any order of the left hand side, thus showing the commutativity of the complete product $\pi(1-\mathscr{T})$.

On the other hand, in Eq. (III-1), the domain of integration can be decomposed into sectors: $0 \leqq \alpha_{i_{1}} \leqq \alpha_{i_{2}}<\cdots<\alpha_{i}$. In each sector, the singularities come from nested subdiagrams $\mathscr{S}_{i}$. The integrand possesses a Laurent series in the dilatation variables of the $\mathscr{S}_{i}^{\prime}$ s. The Taylor operators eliminate the dangerous poles of this series, and ensure the integrability of (III-1).

M. C. Bergère

J. B. Zuber

Service de Physique Théorique

Centre d'Etudes Nucléaires de Saclay

B.P. no. 2

F-91190 Gif-sur-Yvette, France 